\title{
ESTUDIO BIOGEOGRÁFICO DE LAS FUCALES Y LAMINARIALES ATLÁNTICAS EN EL LITORAL MEDITERRÁNEO DE MARRUECOS
}

\author{
Juan Antonio GONZÁLEZ GARCÍA y Francisco CONDE POYALES
}

\begin{abstract}
RESUMEN. Estudio biogeográfico de las Fucales y Laminariales atlánticas en el litoral mediterráneo de Marruecos. La costa mediterránea marroquí, por su proximidad al Estrecho de Gibraltar, presenta en su flora marina elementos cuyo origen y distribución son claramente atlánticos. Se ha estudiado, durante cuatro años, un conjunto de grandes algas pardas (Phaeophyta) con estas características, estableciendo a nivel fitogeográfico los límites de influencia oceánica en dichas costas.
\end{abstract}

Palabras clave. Fucales, Laminariales, Mediterráneo, Marruecos.

ABSTRACT. Bogeographical study of Atlantic Fucales and Laminariales in the Mediterranean Coasts of Morocco. The maroccan mediterranean coast, because of its proximity to the Straits of Gibraltar, presents elements in its seascape flora which are clearly ot atlantic origin. We have been studied a group of big and brown seaweeds (Phaeophyta) with these characteristics for four years, stablishing at a phytogeographic level, the boundaries of the oceanic influence on these coasts.

Key words. Fucales, Laminariales, Mediterranean Sea, Morocco

\section{INTRODUCCIÓN}

En los órdenes Fucales y Laminariales (Phaeophyta) se incluyen un conjunto de especies de gran tamaño y origen fitogeográfico atlántico, cuya presencia en las costas mediterráneas marroquíes y españolas queda limitada a zonas con fuerte influencia oceánica.

En este trabajo hemos realizado un estudio de aquellas especies que, presentando una distribución fundamentalmente atlántico boreal o tropical, penetran en el Mediterráneo através del Estrecho de Gibraltar. La distribución en el Mediterráneo de estas especies puede apoyar una división fitogeográfica del Mar de Alborán en dos zonas, una con acusada influencia atlántica y otra mediterránea. Esta división, basándose en datos obtenidos en el litoral mediterráneo de Andalucía, ha sido anteriormente propuesta por Flores y Conde (1988) y Conde (1989).

\section{MATERIAL Y MÉTODOS}

La zona litoral estudiada abarca desde Punta de Pescadores (Alhucemas) hasta Saidía en las proximidades de la frontera con Argelia 


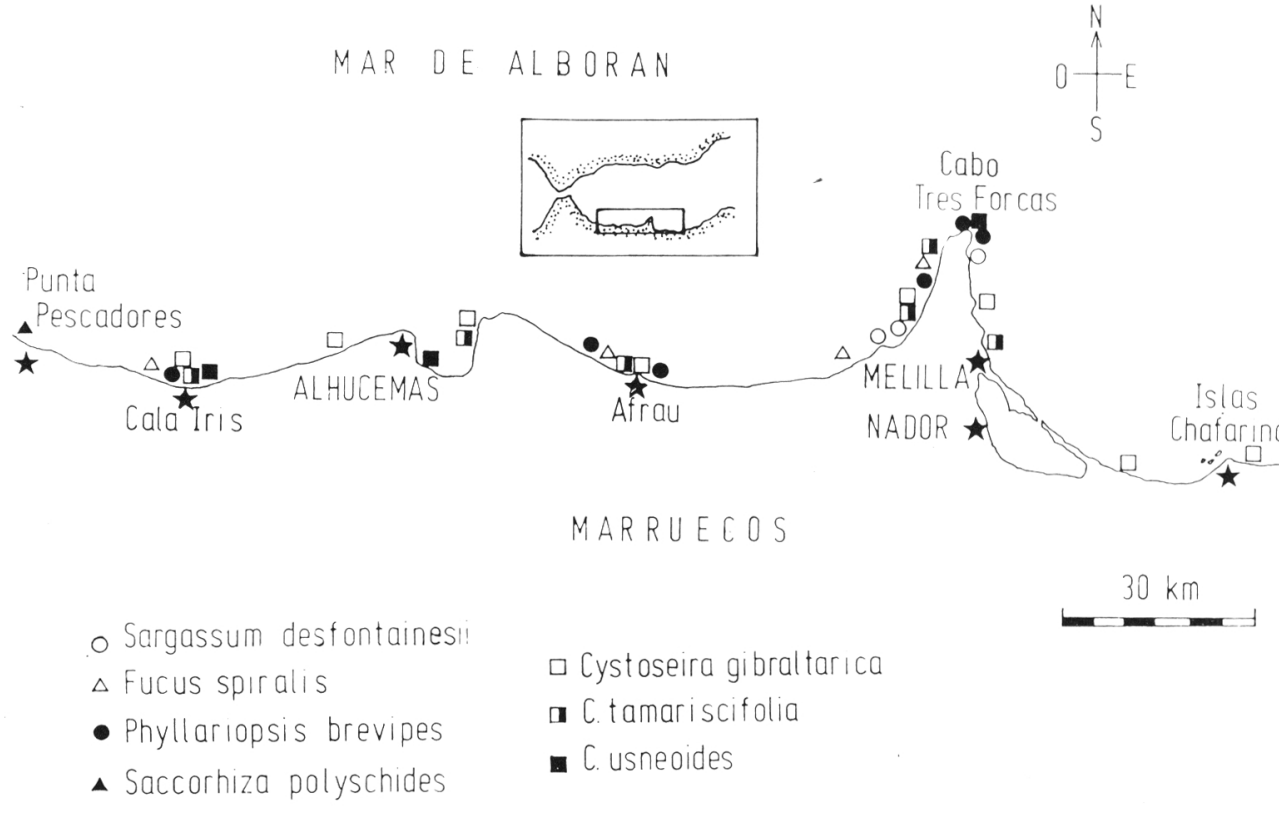

Figura 1. Distribución de taxa de Laminariales y Fucales de procedencia atlántica en el Mediterráneo de Marruecos.

(fig. 1). Se establecieron 28 estaciones de muestreo permanente y se realizaron recolecciones más esporádicas en el resto de la zona; los muestreos se realizaron hasta aproximadamente 30 metros de profundidad.

Con el material recolectado y los datos bibliográficos existentes (Bornet,1892; Debray,1897; Hariot,1909 y 1911; de Buen, 1913; Sauvageau,1913, 1918 y 1920; Bellón,1925; Lemoine,1926 y 1952; Feldmann,1934; Lozano Cabo,1953; Werner,1962; Conde,1984a; Navarro y Gallardo,1989; González-García y Conde,1991 y 1992) elaboramos un catálogo previo indicando para cada una de las especies su posición biogeográfica (A: atlántico; $\mathrm{Ab}$ : atlántico boreal; At: atlántico tropical; M: mediterráneo; P: pantropical) así como referencia bibliográfica concreta para aquellas especies cuya presencia no ha sido confirmada en nuestros muestreos.

Los pliegos testigos de las especies reco- lectadas y tratadas en este trabajo se encuentran depositados en el Herbario del Departamento de Biología Vegetal de la Facultad de Ciencias de la Universidad de Málaga (MGC PHYC).

\section{RESULTADOS}

Las especies citadas o recolectadas en la zona de estudio son las siguientes:

Cystoseira amentacea Bory var. stricta Montagne. (M).

C. baccata (Gmelin) Silva. (A). Chafarinas (Navarro y Gallardo, 1989).

C. brachycarpa J. Ag. emend. Giaccone var. balearica (Sauv.) Giaccone. (M).

C. compressa (Esper) Gerloff et Nizamuddin. (Ab).

C. crinita (Desf.) Bory. (M).

C. elegans Sauv. (M).

C. foeniculacea (L.) Grev. (Ab). Ceuta y 
Melilla. (Werner 1962).

C. gibraltarica (Sauv.) Dangeard. (Ab).

C. humilis Kütz. (Ab).

C. mediterranea Sauv. (M).

C. platyclada Sauv. (M). Melilla (Sauvageau, 1913 y Werner, 1962).

C. sauvageauana Hamel. (M).

C. schiffneri Hamel. (M).

C. spinosa Sauv. (M).

C. tamariscifolia (Huds.) Papenfuss. (Ab).

C. usneoides (L.) Roberts. (Äb).

C. zoosteroides (Turner) J. Ag. (M).

Sargassum acinarium (L.) C. Ag. (At).

S. desfontainesii (Turner) C. Ag. (At).

S. hornschuchii C. Ag. (M).

S. vulgare C. Ag. (P).

Fucus spiralis L. (Ab).

Laminaria rodriguezii Born. (M). Melilla (Sauvageau, 1913 y Werner, 1962).

Phyllariopsis brevipes (C. Ag.) Henry et South. (Ab).

Ph. purpurascens (C. Ag.) Henry et South. (Áb).Melilla (Sauvageau, 1913 y Werner, 1962).

Saccorhiza polyschides (Lightf.) Batters. (Ab).

De la tabla anterior comentaremos sólo aquellas especies que, por ser fundamentalmente de areal atlántico y distribución mediterránea muy restringida, nos pueden indicar condiciones de oceanidad en la zona estudiada. Las especies que aparecen con referencia bibliográfica no han sido recolectadas durante el período de estudio, por lo que dudamos de su presencia en la zona.

\section{Cystoseira gibraltarica (Sauvageau) Dangeard}

Especie de distribución atlántico boreal, se desarrolla en zonas poco profundas, bien iluminadas y aguas más o menos encalmadas.

En Marruecos se encuentra fundamentalmente en la costa atlántica y en el Estrecho de Gibraltar; citada por Sauvageau (1913) en Ceuta, nosotros la hemos recolectado, relativamente abundante, en las estaciones de muestreo más occidentales (fig. 1) y en Cabo de Agua (MGC PHYC: 2119) y Tres Forcas (MGC PHYC: 2123, 2129 y 2135).
No se conocen citas para las costas españolas del Mar de Alborán, ni para otros puntos del Mediterráneo; no obstante existen referencias para las costas mediterráneas de España y Marruecos de C. mauritanica Sauv. (Sauvageau, 1913, 1920), taxon probablemente coespecífico con C. gibraltarica (OliverasPlá y Gómez-Garreta, 1989).

\section{Cystoseira tamariscifolia (Hudson) Papenfuss}

Especie de distribución atlántica boreal que se desarrolla en una franja más o menos contínua entre los niveles litoral e infralitoral, siempre en cantiles rocosos batidos por el oleaje.

Hemos localizado poblaciones de $C$. tamariscifolia en Punta Rostrogordo (Melilla) (MGCPHYC: 2170), Cabo Tres Forcas, Cazaza (MGC PHYC: 2169) y en Punta Afrau, Cabo Quilates y Cala Iris.

En las costas mediterráneas se localiza sólo en las proximidades del Estrecho de Gibraltar, siendo sustituida progresivamente por C. mediterranea. Creemos que las referencias sobre C. tamariscifolia en las costas argelinas (Perret-Boudouresque \& Seridi, 1989), deben corresponder a C. mediterranea .

En el litoral mediterráneo de Marruecos, el Cabo Tres Forcas (fig. 1) es la zona de sustitución de una especie por otra; en la costa mediterránea española esta sustitución tiene lugar a partir de Cabo de Gata, en la provincia de Almería (Soto, 1987). Poblaciones dispersas, fuera del areal anterior, han sido reseñadas para las Islas Chafarinas (Navarro y Gallardo 1989), y para Sicilia (Giaccone et al. 1985).

\section{Cystoseira usneoides (L.) Roberts}

Taxon atlántico boreal, que hemos encontrado comúnmente arrojado o flotando durante el verano en la zona de Cabo de Tres Forcas y en la Bahía de Alhucemas (MGC PHYC: 2172). En Cala Iris (fig. 1) forma densas poblaciones entre $-2 \mathrm{~m}$ a $-6 \mathrm{~m}$, que 
presentan receptáculos de mayo a agosto. Presenta una distribución muy restringida en el Mediterráneo, habiendo sido citada en Ceuta (Bornet,1892), litoral mediterráneo español (Conde, 1984b), Estrecho de Messina, Sicilia (Giaccone et al., 1985) y en Argelia (PerretBoudouresque \& Seridi, 1989).

\section{Sargassum desfontainesii (Turner) C. Ag.}

Taxon de origen atlántico tropical, que en el Mediterráneo se ha localizado exclusivamente en Cabo Tres Forcas (González-García y Conde, 1992).

\section{Fucus spiralis L.}

Especie de origen atlántico boreal, donde forma densas poblaciones en la franja intermareal fotófila, aparece en las costas mediterráneas marroquíes en poblaciones de individuos dispersos y pequeño tamaño (hasta $8 \mathrm{~cm}$ ), sobre rocas horizontales y verticales. La consideramos como el bioindicador florístico más fiable en cuanto a la determinación de la influencia oceánica en el Mar de Alborán.

La hemos localizado en Tres Forcas (MGC PHYC: 2167 y MGC PHYC: 2183), Afrau (MGC PHYC: 2184) y en Cala Iris (MGC PHYC: 2168 y 2181); en esta última localidad coexiste con el endemismo mediterráneo Rissoella verruculosa. Esta especie ha sido anteriormente citada para las costas cercanas a Ceuta por Bornet (1892), Sauvageau (1913) y Werner (1962) y en el litoral mediterráneo francés por Sancholle (1988).

Phyllariopsis brevipes (C. Ag.) Henry et South subsp. brevipes

De origen atlántico boreal, este taxon suele desarrollarse a finales de julio en el infralitoral inferior y circalitoral, formando extensas praderas, de medio metro de altura, sobre fondos horizontales con pequeñas incrustaciones calcáreas, probablemente de Mesophyllum lichenoides, o arenoso y fuertes corrientes marinas. Ha sido citada anterior- mente para la zona de Melilla por Sauvageau (1920) y Werner (1962).

Se ha encontrado a profundidades de $-5 \mathrm{~m}$ (fijada a los hierros oxidados de un barco hundido) y a $-30 \mathrm{~m}$ en diversos puntos del vértice del Cabo Tres Forcas (MGC PHYC: 2163 y 2166). En Cala Charranes (Tres Forcas) se localizó una población a $-1,5 \mathrm{~m}$, eran individuos jóvenes de menos de $5 \mathrm{~cm}$ de tamaño (MGC PHYC: 2180). La hemos localizado tambien en Afrau (MGC PHYC: 2162) y Torres de Alcalá, Cala Iris (MGC PHYC: 2164). La zona de Los Farallones (MGCPHYC: 2165), al oeste de Cabo Tres Forcas, parece ser el límite oriental de esta especie. Ha sido citada en varias localidades del Mediterráneo (España peninsular y balear, Mediterráneo francés, Córcega, Argelia y Sicilia) por Ribera et al. (1992). Suele presentarse fuertemente epifitada por Ectocarpus fasciculatus.

Parte del material (MGC PHYC: 2165) presenta un estipe más largo $(5 \mathrm{~cm})$ que el que describen Pérez-Cirera et al., 1991 para poblaciones del Atlántico (no mayor de $2 \mathrm{~cm}$ ). Puesto que estos autores utilizan este carácter como diferencial entre este tax on y P. brevipes (C. Ag.) Henry et South subsp. pseudopurpurascens Pérez-Cirera, Cremades, Bárbara y López (cuyo estipe oscila entre 2 y $13 \mathrm{~cm}$ ), está claro que las características de diagnosis deberían revisarse.

Saccorhiza polyschides (Lightfoot) Batters Especie de areal atlántico citada en Tres Forcas (de Buen,1913) y confirmada por nosotros, también en Ceuta y Melilla (Werner,1962). La hemos localizado en la zona de Punta de Pescadores (MGC PHYC: 2185) formando extensas praderas, de hasta $2 \mathrm{~m}$ de altura y gran densidad de individuos, entre $-8 \mathrm{~m}$ y $-15 \mathrm{~m}$, desapareciendo, más o menos rápidamente, a profundidades superiores. Estas praderas recubren roca viva subhorizontal, encontrándose poblaciones en distintas fases o estadios de su ciclo vital. Los ejemplares se encuentran 
profusamente epifitados por Ectocarpáceas.

Menos citada en el Mediterráneo que Phyllariopsis brevipes, conocemos recolecciones de Estepona(Málaga) (Conde y Seoane, 1982) y en las proximidades del Estrecho de Messina (Sicilia) y Golfo de Nápoles (Italia) (Ribera et al., 1992). También se halló de arribazón en el litoral de Grecia (Athanasiadis, 1987).

\section{DISCUSIÓN Y CONCLUSIONES}

El Cabo de Tres Forcas parece constituir una barrera geográfica importante para el avance de especies atlánticas en las costas mediterráneas africanas (fig. 1), ya que algunas de ellas no suelen sobrepasarlo o lo hacen de manera muy puntual.

Las grandes laminarias Saccorhiza polyschides y Phyllariopsis brevipes, son características, en el mediterráneo marroquí, de aguas profundas, con fuertes corrientes y poco contaminadas.

Dudamos de la presencia en la zona de Phyllariopsis purpurascens, Cystoseira foeniculacea y Laminaria rodriguezii, y creemos que algunas se tratan de determinaciones incorrectas debido posiblemente a estudiar material muy deteriorado para una correcta identificación (Perret-Boudouresque \& Seridi, 1989; Pérez-Cirera et al., 1991).

Aunque presentes en la zona estudiada hemos excluído como indicadoras de oceanidad a Cystoseira compressa, C. humilis, Sargassum acinarium y $S$. vulgare, pues siendo especies de distribución fundamentalmente atlánticas o pantropicales, están ampliamente distribuidas en el Mediterráneo (Ribera et al., 1992).

Laminaria ochroleuca Pylaie es un taxon no citado por autores anteriores, ni encontrado por nosotros en este litoral, sin embargo, se halla en las costas mediterráneas ibéricas (Málaga), Sicilia y Argelia (Ribera et al., 1992) y también en la Isla de Alborán (Soto y Conde, en prensa).

C. baccata, citada por Navarro y Gallardo (1989) en Chafarinas, es taxon excludenda (Gallardo, com. pers.), estando el material actualmente en estudio.

AGRADECIMIENTOS. A la Dra. GómezGarreta, por la confirmación y revisión de pliegos de Cystoseira y a D. Antonio Flores Moya en los de Phyllariopsis y en la elaboración del mapa. No en último lugar a D. Luis Cruz Sánchez del Club Anfora de Melilla por las numerosas veces que nos acompañó en las inmersiones.

\section{BIBLIOGRAFÍA}

ATHANASIADIS, A. -1987- A survey of the seaweeds of the Aegean Sea. Manuscript. Ph. D. Thesis, University of Gothenburg, Sweden, $174 \mathrm{pp}$.

BELLÓN, L. -1925- Notas sobre una Saccorhiza bulbosa (Hudson) La Pylaie de Melilla (Mediterráneo Occidental) Nuova Notarisia, 40: 217221.

BORNET, E. -1892- Les algues de P.K.A. Schousboe récoltées au Maroc et dans la Méditerranée de 1815 à 1829. Mém. Soc. Nat. Sci. Nat. et Mathémat. Cherboug, 28: 165-376.

BUEN, O., DE -1913- Note sur les fonds et sur la pêche dans la côte méditerranéenne du Riff. Atti $5^{\circ}$ Congresso Internazionale di Pesca. Rome.

CONDE, F. - 1984a- Contribución al conocimiento de la flora bentónica del Mar de Alborán. Islas Chafarinas. Acta Bot. Malacitana, 9: 41-46.

CONDE F. -1984b- Catálogo de las algas macrobentónicas marinas de Málaga. Acta Bot. Malacitana, 9: 47-78.

CONDE, F. -1989- Ficogeografía del Mar de Alborán en el contexto del Mediterráneo Occidental. An. Jard. Bot. Madrid, 46 (1): 21-26.

CONDE, F. y J. SEOANE -1982- Aspectos de la vegetación y zonación macrofitobentónica en las costas malagueñas. An. Jard. Bot. Madrid, 39 (2): 465-487. 
DEBRAY, A. -1987- Catalogue des algues du Maroc, d'Algerie et de Tunisie. Librairie A. Jourdan. Alger. 78 pp.

FELDMANN, J. -1934- Les Laminariacées de la Méditerranée et leur répartition géographique. Bull. Trav. Stat. d'Aquicult. et Pêche de Castiglione, 2: 3-42.

FLORES, A. y F. CONDE -1988-Importancia del Estrecho de Gibraltar en el estudio de la biogeografía de los macrófitos bentónicos del Mar de Alborán: nuevas contribuciones. Actas del Congreso Internacional: El Estrecho de Gibraltar, Ceuta, 425-432.

GIACCONE, G., P. COLONNA, C. GRAZIANO, A. MANNINO, E. TORNATORE, M. CORMACI, G. FURNARI \& B. SCAMACCA -1985-Revisione della flora marina di Sicilia e isole minori. Boll. Acc. Gioenia Sci. Nat., 18(326): 535-781.

GONZÁLEZ GARCÍA, J.A. y F. CONDE -1991Estudio florístico, fenológico, autoecológico y fitogeográfico del macrofitobentos de la Mar Chica (Sebcha Buareg de Nador, Mediterráneo marroquí). Acta Bot. Malacitana, 16(1): 63-80.

GONZÁLEZ GARCÍA, J.A. y F. CONDE -1992Sargassum desfontainesii (Turner) C. Agardh (Fucales, Fucophyceae), nueva especie para el Mediterráneo. Acta Bot. Malacitana, 17: 250251.

HARIOT, M.P. -1909- Sur une collection d'algues recueillies à Maroc par M. Buchet. Bull. Mus. Hist. Nat., 3: 128-130.

HARIOT, M.P. -1911- Sur une collection d'algues recueillies à Mauritania par M. Chudeau. Bull. Soc. Bot. Fr., 58: 438-445.

LEMOINE, P. -1926- Corallinacées du Maroc II. Bull. Soc. Sc. Nat. Maroc, 6(1-6): 106-108.

LEMOINE, P. -1952- Corallinacées de France et d'Afrique du Nord. Arch. Mus. Hist. Nat. Tome I: $15-136+23$ pl.

LOZANO CABO, F. -1953- Notas sobre una prospección pesquera en la Mar Chica. Bol. Inst. Esp. Ocean., 64: 3-37.

NAVARRO, M.J. y T. GALLARDO -1989- Aportación al conocimiento de la flora bentónica de las costas mediterráneas africanas occidentales. Bot. Complutensis, 15: 203-214.

OLIVERAS PLA, M.A. y A. GOMEZ GARRETA -1989- Corología del género Cystoseira C. Agardh (Phaeophyceae, Fucales). An. Jard.
Bot. Madrid, 46(1): 89-97.

PEREZ-CIRERA, J.L., J. CREMADES, I. BARBARA y M.C. LOPEZ -1991- Contribución al conocimiento del género Phyllariopsis (Phyllariaceae, Phaeophita) en el Atlántico Europeo. Nova Acta Cientifica Compostelana (Bioloxia), 2: 3-11.

PERRET-BOUDOURESQUE, M. \& H. SERIDI 1989-Inventaire des algues marines benthiques d'Algerie. GIS Posidonie publ., Marseille, Fr., 1-117.

RIBERA, M.A., A. GOMEZ GARRETA, T. GALLARDO, M. CORMACI, G. FURNARI \& G. GIACCONE-1992-Checklist of Mediterranean Seaweeds. I. Fucophyceae. Botanica Marina, 35: 109-130.

SANCHOLLE, M. -1988- Présence de Fucus spiralis (Phaeophyceae) en Méditerranée Occidentale. Cryptogamie, Algol., 9: 157-161.

SAUVAGEAU, C. -1913- Sur les fucacées du étroit de Gibraltar. Comp. Rend. Hebd. Séances Acad. Sci., 157: 1539-1540.

SAUVAGEAU, C. -1918- Recherches sur les Laminaires des côtes de France. Mem. Acad. Sc., 56: 1-240.

SAUVAGEAU, C. -1920- A propos des Cystoseira de Banyuls et de Guétary. Bull. Stat. Biol. Arcachon, 17: 3-51.

SOTO, J. -1987- Estudio florístico, corológico, autoecológico y sinecológico de las algas bentónicas marinas del sureste de la Península Ibérica. Tesis Doctoral. Universidad de Málaga. $507 \mathrm{pp}$.

SOTO, J. y F. CONDE (en prensa) Datos sobre la flora algal bentónica de la Isla de Alborán (Mar de Alborán, Mediterráneo Occidental). Cryptogamie, Algol.

WERNER, R.G. -1962- Essai d'une étude de la repartition des Cryptogames marines et maritimes du Maroc. Bull. Soc. Sci. Nat. \& Phys. Maroc, 42: 1-33.

Aceptado para su publicación en Junio de 1993

Dirección de los autores. J.A. González: Escuela Universitaria de Formación del Profesorado. Melilla. F. Conde: Departamento de Biología Vegetal. Facultad de Ciencias. Universidad de Málaga. 